OPEN ACCESS

Edited by:

Akira Sugawara,

Tohoku University, Japan

Reviewed by:

Masha Livhits,

UCLA David Geffen School of Medicine, United States

Daniele Barbaro,

UO Endocrinologia ASL nord ovest

Toscana, Italy

Fumihiko Furuya,

University of Yamanashi, Japan

*Correspondence:

Wei Cai

caiwei@shsmu.edu.cn

Weihua Qiu

qwh11072@rih.com.cn

Lei Tao

doctortaolei@163.com

${ }^{\dagger}$ These authors have contributed equally to this work

Specialty section:

This article was submitted to

Thyroid Endocrinology,

a section of the journal

Frontiers in Endocrinology

Received: 19 July 2021 Accepted: 08 September 2021 Published: 30 September 2021

Citation:

Yang Z, Heng Y, Zhao Q, Cao Z, Tao L,

Qiu W and Cai W (2021) A Specific Predicting Model for Screening Skip Metastasis From Patients With Negative Central Lymph Nodes Metastasis in Papillary Thyroid Cancer.

Front. Endocrinol. 12:743900. doi: 10.3389/fendo.2021.743900

\section{A Specific Predicting Model for Screening Skip Metastasis From Patients With Negative Central Lymph Nodes Metastasis in Papillary Thyroid Cancer}

\author{
Zheyu Yang ${ }^{1+}$, Yu Heng ${ }^{2 \dagger}$, Qiwu Zhao ${ }^{1+}$, Zichao Cao ${ }^{1}$, Lei Tao ${ }^{2 *}$, Weihua Qiu ${ }^{1 *}$ \\ and Wei Cai ${ }^{1 *}$ \\ ${ }^{1}$ Department of General Surgery, Ruijin Hospital, Shanghai Jiao Tong University School of Medicine, Shanghai, China, \\ ${ }^{2}$ ENT Institute and Department of Otorhinolaryngology, Eye \& ENT Hospital, Fudan University, Shanghai, China
}

Skip metastasis is a specific type of papillary thyroid cancer lymph node metastasis (LNM). The present study aimed to clarify the typical clinical characteristics of skip metastasis and optimize the prediction model, so as to provide a more individual treatment mode for skip metastasis. We retrospectively analyzed 1075 PTC patients with different lymph node metastasis statuses from two clinical centers. Comparisons have been made between patients with skip metastasis and other types of LNM. Univariate and multivariate analyses were performed to detect the risk factors for skip metastasis with negative LNM, and a nomogram for predicting skip metastasis was established. The rate of skip metastasis was 3.4\% (37/1075). Compared with other types of LNM, significant differences showed in tumor size, upper portion location, thyroid capsular invasion, and ipsilateral nodular goiter with the central lymph node metastasis (CLNM) group, and in age and gender with the lateral lymph node metastasis (LLNM) group. Four variables were found to be significantly associated with skip metastasis and were used to construct the model: thyroid capsular invasion, multifocality, tumor size $>1 \mathrm{~cm}$, and upper portion. The nomogram had good discrimination with a concordance index of 0.886 (95\% confidence interval [Cl], 0.823 to 0.948). In conclusion, the significant differences between skip metastasis and other types of LNM indicated that the lymph node drainage pathway of skip metastasis is different from either CLNM or LLNM. Furthermore, we established a nomogram for predicting risk of skip metastasis, which was able to effectively predict the potential risk of skip metastasis in patients without preoperative LNM clue.

Keywords: predicting, nomogram, N1b metastasis, papillary thyroid cancer, skip metastasis

\section{INTRODUCTION}

Papillary thyroid cancer (PTC), accounting for $90 \%$ of thyroid cancer pathologies, is the most common type of cancer of the head and neck region, and its incidence is increasing worldwide (1-3). Although the mortality of PTC has not changed, and disease-specific mortality at 10 years is less than $5 \%(4,5)$, the clinical manifestations of PTC patients are not always favorable (6). According to American 
Thyroid Association (ATA) in 2015, risk stratification, capsular invasion, extrathyroidal extension, lymph node metastasis (LNM), incomplete resection, distant metastasis, and vascular invasion are significant risk factors of tumor recurrence or progression $(7,8)$.

In different reports, PTC is shown to involve cervical lymph node metastasis in $20-50 \%$ of patients with macrometastasis and in up to $90 \%$ of patients with micrometastasis detected by sensitive detection methods (9-11). LNM of PTC occurs in a stepwise fashion. Spreading from the thyroid gland, the central and lateral lymph node compartments on the ipsilateral side of the thyroid tumor represent the first echelons of lymphatic drainage followed by the mediastinal and contralateral lateral lymph node compartments $(12,13)$. According to the American Joint Committee on Cancer (AJCC, 8th edition) staging system, LNM is divided into N1a (central lymph node metastasis, CLNM) and N1b (lateral lymph node metastasis, LLNM) (14).

Skip metastases, which leaps over the central compartment, is not uncommon in cases of PTC with lateral cervical lymph node metastasis. The frequency of skip metastasis has been reported to vary between $7.4 \%$ and $21.8 \%$ in PTC with lateral LNM (15-17). Skip metastases have also been reported in other tumors; the prognosis of skip metastases in different tumors is also significantly different (18-20). Previous studies have constructed several prediction models for the risk of skip metastasis, identifying patients with skip metastasis from that with LLNM, which had limited clinical value $(15,16,21,22)$. The purpose of the present study is to precisely describe the clinical characteristics of skip metastasis on the basis of previous studies and build a prediction model with more clinical significance.

\section{METHODS}

\section{Patient Cohort}

Between June 2017 and June 2019, 1179 newly diagnosed primary PTC patients who underwent thyroidectomy at Department of General Surgery, Ruijin Hospital, Shanghai Jiao Tong University School of Medicine, and Department of Otorhinolaryngology, Head and Neck Surgery, at the Eye, Ear, Nose, and Throat (EENT) Hospital of Fudan University were enrolled in our study. All patients included in the study had pathologically confirmed PTC, and we excluded patients with poorly differentiated papillary cancer (PDTC). After exclusion of patients with no lymph nodes removed $(n=33)$ or incomplete laboratory and pathological results $(n=71), 1,075$ patients who had pathological PTC and received thyroidectomy along with lymph node dissection were studied. 1075 patients included 381 males (35.4\%) and 694 females (64.6\%), with a mean age of 42.9 (18-71) years, and mean BMI of 23.81 (16.90-40.52) (Table 1). None of the patients had existing distant metastasis or other malignancies when diagnosed and all of them were confirmed as PTC by postoperative pathology.

\section{Data Collection, Surgical Procedure, and Pathological Approach}

Preoperative data sources including serum index, ultrasound examination (US) and fine-needle aspiration (FNA) were
TABLE 1 | Demographics and clinical Characteristics of the cohort.

\begin{tabular}{lc}
\hline Variable & Value (\%) \\
\hline Age & \\
mean & \\
BMI & 42.9 \\
mean & \\
Gender & 23.73 \\
male & \\
female & $381(35.4)$ \\
Size of largest lesion (US) & $694(64.6)$ \\
mean & \\
medium & 0.93 \\
Thyroid capsule invasion (US) & 0.9 \\
Multifocality (US) & $435(40.5)$ \\
Bilateral disease & $332(30.9)$ \\
Negative LNM & $236(21.9)$ \\
mean harvested CLN & $495(46.1)$ \\
LNM & 5.1 \\
Skip metastasis & $580(53.9)$ \\
$\quad$ mean harvested CLN & $37(6.4)$ \\
CLNM & 7.9 \\
mean harvested CLN & \\
mean positive CLN & $383(66)$ \\
$\quad$ mean harvested CLN & 6.7 \\
mean positive CLN & 3.3 \\
\hline & $160(27.6)$ \\
\hline & 8.1 \\
LNM & 4.1 \\
\hline
\end{tabular}

collected from Electronic Medical Records System for further analysis. Preoperative US and US-guide FNA were performed strictly according to Thyroid Imaging Reporting and Data System (TI-RADS), Preoperative US provided standardized description of the number of tumors, tumor location, tumor size, tumor edge distance from capsule( $<0 \mathrm{~mm}$ defined as capsular invasion), and lymph nodes involved. In addition to thyroid and parathyroid glands, a description of central and lateral lymph nodes was also included. All patients enrolled were identified as T0-4N0-1bM0 according to the 2015 American Joint Committee on Cancer (AJCC) Tumor Node Metastasis (TNM) staging system.

The basic surgical procedure consisted of a total lobectomy with a central compartment lymph node dissection (LND). The nodal tissue within the central compartment was removed from the level of the hyoid bone along the recurrent laryngeal nerves (RLN), and to a comparable level behind the clavicle. All resected tissues and lymph nodes were sent for intraoperative pathology examinations. For those with CLNM, bilateral thyroidectomy was performed simultaneously, but no elective lateral neck dissections were performed. Routine exploration of the ipsilateral neck was conducted for suspected level II, III, IV, and V lymph node metastasis.

All acquired specimens were examined by two or more boardcertified pathologists from Shanghai Ruijin Hospital \& Shanghai EENT Hospital. Pathological features analyzed were pathological type of tumor, type of the surrounding thyroid tissues, tumor size, capsular invasion, extrathyroidal extension, multifocality (more than one lesion in unilateral thyroid lobe), and lymph node metastasis.

\section{Statistical Analysis}

Chi-square test and independent t-test were conducted for categorical variables and continuous variables respectively. 
Univariate and multivariate analyses were conducted for screening risk variables that were significantly associated with the skip metastases. $\mathrm{P}<0.05$ was considered to indicate a statistically significant difference, and statistical analyses were conducted using the SPSS 24.0 package (SPSS Inc., Chicago, IL, USA). Variables with the p-value $<0.05$ from the univariate logistic regression were then used for multivariate logistic regression to construct a risk prediction model - Nomogram, in $\mathrm{R}$ software (ver. 3.5.1, $\mathrm{R}$ Development Core Team). The discrimination and consensus degree of our newly-established predictive model were tested through the receiver operating characteristic (ROC) curve, the calibration curve, and the concordance index (C-index).

\section{Ethical Statement}

This study was approved by the Institutional Ethics Committee of the Eye and ENT Hospital of Fudan University and Ruijin Hospital, Shanghai Jiao Tong University School of Medicine, and was also approved by Chinese Clinical Trial (ChiCTR2100043353). All participants gave informed consent to take part in the study after full explanation of the purpose and nature of all procedures used.

\section{RESULTS}

\section{Demographics and Clinicopathological Characteristics of Patients in the Cohort}

The characteristics of the study patients are given in Table $\mathbf{1}$. Through preoperative US detection and fine needle aspiration biopsy (FNA), the mean tumor size was $0.93 \mathrm{~cm}$ with a range of 0.05-5.4 cm. Of the patients, $435(40.5 \%)$ were diagnosed with thyroid capsule invasion, 332 (30.9\%) were detected with multifocality, $236(21.9 \%)$ were confirmed to have bilateral PTC, and 580 (53.9\%) were ultimately confirmed to have LNM by postoperative pathology. In patients with LNM, 37 patients (6.4\%) were diagnosed with skip metastasis, while 383 (66\%) with CLNM and 160 (27.6\%) with LLNM. The mean harvested central lymph nodes (CLN) in skip, CLNM, and LLNM were 7.9, 6.7, and 8.1, respectively, and the mean positive CLN in CLNM and LLNM were 3.3 and 4.1, respectively.

\section{The Comparison Between Patients With Skip Metastasis and Conventional Pathway of Metastasis (CLNM Only and CLNM Accompanied With Lateral Cervical Lymph Node Metastasis)}

$580(54.0 \%)$ patients were confirmed to have positive cervical lymph node metastases in our cohort. We classified them into three subgroups according to the extent of neck metastases: Skip Group (patients with skip metastasis, $n=37$ ), CLNM Group (patients with CLNM only, $\mathrm{n}=383$ ), and LLNM Group (patients with both CLNM and LLNM, $n=160$ ). Patients in Skip Group showed significantly larger tumor size than those in CLNM Group (P-value $=0.002)$, however, no such difference was found between Skip Group and LLNM Group (P-value>
0.05). More patients exhibited thyroid capsular invasion and ipsilateral nodular goiter in Skip Group than in CLNM Group (P-value $=0.004$ and 0.022 , respectively). As for tumor location, significant difference was found between Skip Group and CLNM Group: upper pole tumors were more common in Skip Group, and lower pole ones were relatively fewer. But no difference was shown between Skip Group and LLNM Group (P-value= 0.275). Moreover, a difference also existed between Skip Group and LLNM Group in age and gender (P-value $=0.009$ and 0.018, respectively) (Table 2 ).

\section{Univariate and Multivariate Logistic Regression for Identifying Independent Risk Factors of Skip Metastasis in Patients With No Central Lymph Node Metastasis}

Main clinicopathological characteristics of patients with no CLNM were shown in Table 3. Significantly more patients exhibited thyroid capsular invasion $(\mathrm{P}$-value $=0.000)$ and bilateral disease $(\mathrm{P}$-value $=0.025)$ in Skip Group than in Negative LNM Group, and multifocality was also more common in Skip Group $(\mathrm{P}$-value $=0.000)$. Factors including age $>40$, gender, PTC with ipsilateral Hashimoto thyroiditis, and nodular goiter all showed no significant difference between these two subgroups (P-value> 0.05). In all patients with no central lymph node metastasis, the presence of thyroid capsular invasion, bilateral disease and multifocality, tumor in the upper portion, and maximum tumor diameter $>=1 \mathrm{~cm}$ are significantly associated with skip metastasis in the univariate analysis. Those elements were then enrolled into the multivariate logistic regression analysis. As a result, a total of four variables were confirmed as independent risk factors of skip metastasis in patients with no central lymph node metastasis: thyroid capsular invasion, multifocality, tumor in the upper portion, and maximum tumor diameter $>=1 \mathrm{~cm}$ (Table 4).

\section{NOMOGRAM FOR PREDICTING PROBABILITY OF SKIP METASTASIS AND VALIDATION OF THE NOMOGRAM}

Based on the independent factors screened through multivariate analysis, a nomogram was established for predicting individual risk of skip metastasis. The risk of each factor, including thyroid capsular invasion (TCI), maximum tumor diameters (MTD), multifocality, and upper portion, was quantified in our predicting model (shown in Figure 1) to predict the presence of skip metastasis from patients with negative LNM. For evaluating our nomogram's ability to predict skip metastasis in PTC patients, we conducted an internal validation using 1,000 bootstrap resamples. As a result, C-index was found to be 0.886 (95\% CI, 0.823 to 0.948 ), and 0.888 (95\% CI, 0.827-0.948) after bootstrapping (Figure 2A). Furthermore, we also conducted a calibration plot for our newly-established model, and a favorable agreement was shown between the actual and estimated probability of skip metastasis (Figure 2B). 
TABLE 2 | The clinicopathological characteristics of PTC patients with LNM.

\begin{tabular}{|c|c|c|c|c|c|c|c|c|}
\hline & \multicolumn{2}{|c|}{ CLNM Group } & \multirow{2}{*}{$\begin{array}{c}\text { P value } \\
\text { (Skip vs. CLNM) }\end{array}$} & \multicolumn{2}{|c|}{ Skip Group } & \multirow{2}{*}{$\begin{array}{c}\text { P value } \\
\text { (Skip vs. CLNM) }\end{array}$} & \multicolumn{2}{|c|}{ LLNM Group } \\
\hline & $n=383$ & $\%$ & & $\mathrm{n}=37$ & $\%$ & & $n=160$ & $\%$ \\
\hline Age & & & 0.222 & & & 0.009 & & \\
\hline$>40$ & 167 & 43.6 & & 20 & 54.1 & & 50 & 31.3 \\
\hline$<=40$ & 216 & 56.4 & & 17 & 45.9 & & 110 & 68.8 \\
\hline Gender & & & 0.192 & & & 0.018 & & \\
\hline Male & 156 & 40.7 & & 11 & 29.7 & & 82 & 51.2 \\
\hline Female & 227 & 59.3 & & 26 & 70.3 & & 78 & 48.8 \\
\hline Maximum tumor diameter (mean \pm SD) & \multicolumn{2}{|c|}{$0.94 \pm 0.62$} & 0.002 & \multicolumn{2}{|c|}{$1.29 \pm 0.84$} & 0.180 & \multicolumn{2}{|c|}{$1.54 \pm 1.09$} \\
\hline Thyroid capsular invasion & & & 0.004 & & & 0.421 & & \\
\hline No & 178 & 46.5 & & 8 & 21.6 & & 45 & 28.1 \\
\hline Yes & 205 & 53.5 & & 29 & 78.4 & & 115 & 71.9 \\
\hline Bilateral disease & & & 0.397 & & & 0.454 & & \\
\hline Absent & 293 & 76.5 & & 26 & 70.3 & & 102 & 63.7 \\
\hline Present & 90 & 23.5 & & 11 & 29.7 & & 58 & 36.3 \\
\hline Multifocality & & & 0.884 & & & 0.190 & & \\
\hline Absent & 223 & 58.2 & & 22 & 59.5 & & 76 & 47.5 \\
\hline Present & 160 & 41.8 & & 15 & 40.5 & & 84 & 52.5 \\
\hline Tumor location & & & 0.018 & & & 0.275 & & \\
\hline Isthmus & 24 & 6.3 & & 1 & 2.7 & & 3 & 1.9 \\
\hline Upper portion & 97 & 25.3 & & 19 & 51.4 & & 62 & 38.8 \\
\hline Middle portion & 102 & 26.6 & & 6 & 16.2 & & 40 & 25.0 \\
\hline Lower portion & 155 & 40.5 & & 11 & 29.7 & & 43 & 26.9 \\
\hline Diffuse TC & 5 & 1.3 & & 0 & 0.0 & & 12 & 7.5 \\
\hline PTC with ipsilateral Hashimoto thyroiditis & & & 0.182 & & & 0.570 & & \\
\hline No & 307 & 80.2 & & 33 & 89.2 & & 137 & 85.6 \\
\hline Yes & 76 & 19.8 & & 4 & 10.8 & & 23 & 14.4 \\
\hline PTC with ipsilateral nodular goiter & & & 0.022 & & & 0.486 & & \\
\hline No & 293 & 76.5 & & 22 & 59.5 & & 85 & 53.1 \\
\hline Yes & 90 & 23.5 & & 15 & 40.5 & & 75 & 46.9 \\
\hline
\end{tabular}

Bold: $P<0.05$.

\section{DISCUSSION}

Skip metastasis to the lateral lymph nodes compartment, which leaps over the central compartment, was found in $7.4 \%$ to $19 \%$ of cases of PTC with LLNM, and researchers have focused on skip metastasis and studied the risk factors of its occurrence in several previous studies $(13,15-17)$. Although relevant studies proposed clinical features of skip metastasis and provided preliminary prediction models, they have common shortcomings in clinical significance and application potential. In the present study, we conducted comparative analysis of skip metastasis to describe its clinical characteristics more comprehensively. In addition to exploring the differences between patients with skip metastasis and other types of lymph node metastases to find the typical characteristics, we further constructed a specific prediction model focusing on screening present and potential skip metastasis from patients with negative CLNM, which might have more potentiality in clinical application.

We compared and analyzed group skip with other types of LNM in order to study the differences in clinical characteristics between group skip and group CLNM/LLNM. Significant differences in maximum tumor diameter, capsular invasion, tumor location, and ipsilateral nodular goiter between group skip and group CLNM, and in age and gender between group skip and group LLNM, were found. Compared with group CLNM, group skip has relatively similar clinical characteristics with group LLNM, however, this does not mean that skip metastasis and LLNM can be simply classified as the same type of LNM. In another multi-center clinical research with prognostic data, we found that the tumor recurrence survival rate of skip metastasis was significantly different from that of LLNM, which was demonstrated for the first time in PTC. Qiu et al. (23) indicated that skip metastasis might be related to less aggressive PTC and better prognosis in their systematic review and metaanalysis. Recognizing the above clinical characteristics is of great help to clarify the cause of skip metastasis and the lymph node metastasis pattern of PTC.

Moreover, we found that skip metastasis and LLNM both have obvious characteristics of upper pole tumor origin. Although there is no significant difference between skip metastasis and LLNM in terms of tumor location (Table 3), we found that patients with skip metastasis have a larger proportion of tumor located in the upper pole of the thyroid gland. Previous studies have reported this characteristic of skip metastasis (13, $22,24)$; this is related to the special lymph node drainage pathway of the thyroid gland, which means that the first cite of lymph node drainage in a different area of the thyroid may be different. From this perspective, although PTC patients with skip metastasis and LLNM have the same characteristic of upper pole tumors, they have different clinical significance and prognostic characteristics. According to the 8th AJCC TNM staging system (14), patients with lateral lymph node metastasis (including skip 
TABLE 3 | The clinicopathological characteristics of PTC patients with no CLNM.

\begin{tabular}{|c|c|c|c|c|c|}
\hline & \multicolumn{2}{|c|}{ Negative LNM Group } & \multicolumn{2}{|c|}{ Skip Group } & \multirow[t]{2}{*}{$P$ value } \\
\hline & $\mathrm{n}=495$ & $\%$ & $\mathrm{n}=37$ & $\%$ & \\
\hline Age & & & & & 0.418 \\
\hline$>40$ & 301 & 60.8 & 20 & 54.1 & \\
\hline$<=40$ & 194 & 39.2 & 17 & 45.9 & \\
\hline Gender & & & & & 0.685 \\
\hline Male & 132 & 26.7 & 11 & 29.7 & \\
\hline Female & 363 & 73.3 & 26 & 70.3 & \\
\hline Maximum tumor diameter(mean $\pm S D$ ) & \multicolumn{2}{|c|}{$0.69 \pm 0.55$} & \multicolumn{2}{|c|}{$1.29 \pm 0.84$} & 0.000 \\
\hline Thyroid capsular invasion & & & & & 0.000 \\
\hline No & 409 & 82.6 & 8 & 21.6 & \\
\hline Yes & 86 & 17.4 & 29 & 78.4 & \\
\hline Bilateral disease & & & & & 0.025 \\
\hline Absent & 418 & 84.4 & 26 & 70.3 & \\
\hline Present & 77 & 15.6 & 11 & 29.7 & \\
\hline Multifocality & & & & & 0.000 \\
\hline Absent & 422 & 85.3 & 22 & 59.5 & \\
\hline Present & 73 & 14.7 & 15 & 40.5 & \\
\hline Tumor location & & & & & 0.021 \\
\hline Isthmus & 29 & 5.9 & 1 & 2.7 & \\
\hline Upper portion & 136 & 27.5 & 19 & 51.4 & \\
\hline Middle portion & 174 & 35.2 & 6 & 16.2 & \\
\hline Lower portion & 148 & 29.9 & 11 & 29.7 & \\
\hline Diffuse PTC & 8 & 1.6 & 0 & 0.0 & \\
\hline PTC with ipsilateral Hashimoto thyroiditis & & & & & 0.131 \\
\hline No & 390 & 78.8 & 33 & 89.2 & \\
\hline Yes & 105 & 21.2 & 4 & 10.8 & \\
\hline PTC with ipsilateral nodular goiter & & & & & 0.095 \\
\hline No & 358 & 72.3 & 22 & 59.5 & \\
\hline Yes & 137 & 27.7 & 15 & 40.5 & \\
\hline
\end{tabular}

Bold: $P<0.05$

TABLE 4 | Univariate and multivariate logistic analyses of skip LNM for PTC patients with no CLNM.

\begin{tabular}{|c|c|c|c|c|}
\hline & \multicolumn{2}{|c|}{ Univariate analysis } & \multicolumn{2}{|c|}{ Multivariate analysis } \\
\hline & Hazard ratio $(95 \% \mathrm{Cl})$ & $P$ value & Hazard ratio $(95 \% \mathrm{Cl})$ & $P$ value \\
\hline \multicolumn{5}{|l|}{ Factors selected } \\
\hline Age & & 0.419 & & \\
\hline$>40$ vs. $<=40$ & $0.758(0.388-1.484)$ & & & \\
\hline Gender & & 0.685 & & \\
\hline Male vs. Female & $1.163(0.559-2.420)$ & & & \\
\hline BMI & & 0.797 & & \\
\hline$>=23.0$ vs. $<23.0$ & $0.916(0.470-1.788)$ & & & \\
\hline Thyroid capsular invasion & & 0.000 & & 0.000 \\
\hline Yes vs. No & $17.240(7.619-39.007)$ & & $12.456(5.304-29.247)$ & \\
\hline Location & & 0.003 & & 0.013 \\
\hline Upper portion vs. non-upper portion & $2.786(1.420-5.468)$ & & $2.736(1.242-6.028)$ & \\
\hline Maximum tumor diameter & & 0.000 & & 0.001 \\
\hline$>=1.0 \mathrm{~cm}$ vs. $<1.0 \mathrm{~cm}$ & $6.096(3.048-12.191)$ & & $4.026(1.820-8.908)$ & \\
\hline Bilateral disease & & 0.029 & & 0.332 \\
\hline Present vs. Absent & $2.297(1.090-4.841)$ & & $1.573(0.629-3.934)$ & \\
\hline Multifocality & & 0.000 & & 0.008 \\
\hline Present vs. Absent & $3.941(1.954-7.951)$ & & $3.110(1.345-7.187)$ & \\
\hline PTC with ipsilateral Hashimoto thyroiditis & & 0.140 & & \\
\hline Yes vs. No & $0.450(0.156-1.299)$ & & & \\
\hline PTC with ipsilateral nodular goiter & & 0.099 & & \\
\hline Yes vs. No & $1.782(0.898-3.535)$ & & & \\
\hline
\end{tabular}

Bold: $P<0.05$ 


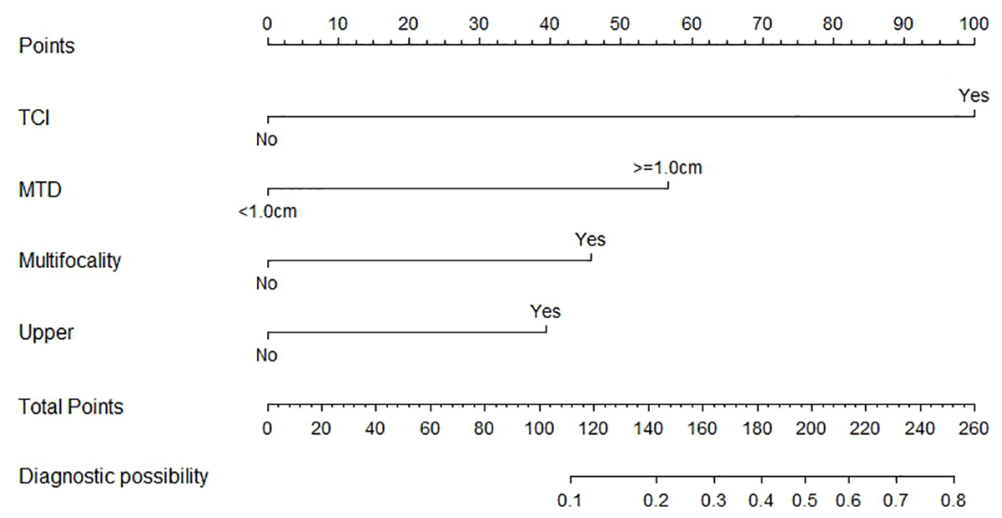

FIGURE 1 | The nomogram for predicting risk of skip metastasis in PTC patients.

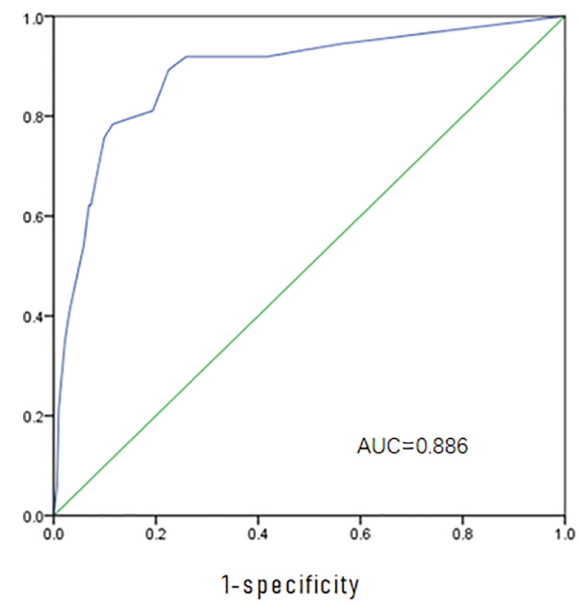

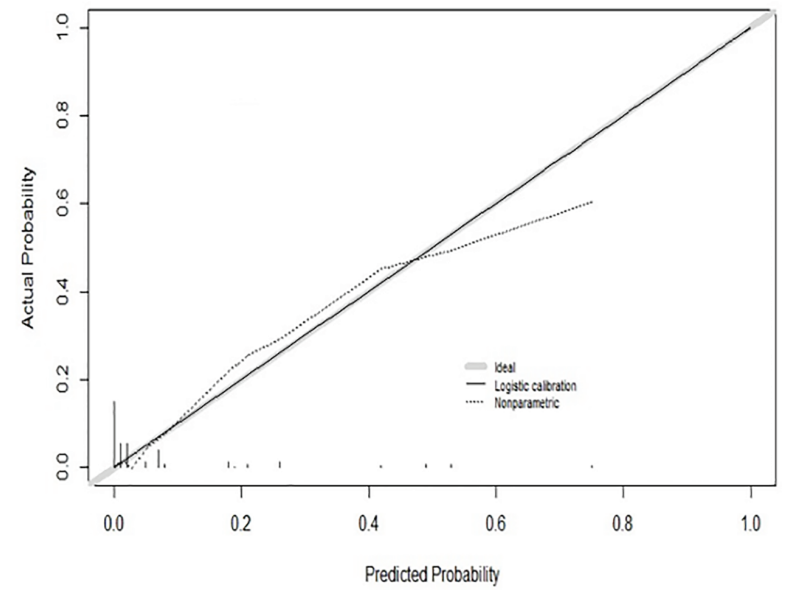

FIGURE 2 | Evaluation and Validation of the nomogram. (A) The ROC curve and AUC of the nomogram; ROC, receiver operating characteristics; (B) The calibration curve of the nomogram for predicting possible CNM. Actual probability is plotted on the $y$-axis, and nomogram predicted probability on the $\mathrm{x}$-axis.

metastasis) should be classified as N1b, however, whether skip metastasis should be simply classified as N1b remains to be further studied.

Separately from the previous studies, we performed univariate and multivariate analysis between group skip and group negative LNM. Predicting metastasis rather than skip, in skip metastasis, was the reason that the assessment model could make sense in clinical practice. Researchers tended to compare and analyze the skip group with the LLNM group $(15,16,24,25)$, while the predicting models did not have specific predictive values, because of the same surgical procedure and treatment strategy for skip metastasis or LLNM patients. However, identifying the present and potential skip metastasis patients from patients without preoperative lymph node metastasis features might provide more individual treatment strategies, including prophylactic lymph node exploration, Postoperative ${ }^{131}$ I treatment, and closer follow-up on lymph nodes. In this study, capsular invasion, primary tumor in the upper portion, maximum tumor diameter $>1 \mathrm{~cm}$, and multifocality are independent risk factors for the occurrence of skip metastasis. The risk factors we obtained were different from the results in previous studies on skip metastasis, but closer to the predicting models for CLNM/ LLNM (26-28). Therefore, we established a new nomogram to evaluate and quantify the risk of skip metastasis. In addition to confirming the patients with definite preoperative metastasis, more importantly, patients with high-risk characteristics of skip metastasis, who may have occult metastasis with negative LNM by preoperative ultrasound, were screened out. 
In this research, we thoroughly studied the risk factors and clinical characteristics of skip metastasis. Compared with PTC patients with negative lymph node metastasis, patients with characteristics of capsular invasion, primary tumor in the upper portion, maximum tumor diameter $>1 \mathrm{~cm}$, and multifocality are more likely to suffer skip metastasis. As a result, we could screen out the at-risk patients of skip metastasis from the negative LNM group, which played an important role in making an individual surgical option, reducing the incidence of secondary operations, formulating accurate active surveillance strategy, etc. At the same time, by comparing the similarities and differences between skip metastasis and other types of LNM, we found that tumor location played an important role in skip metastasis, which led to conjectures on the unique lymphatic metastasis pathway of skip metastasis. To sum up, we have made a comprehensive observation and analysis of skip metastasis, explaining its causes, drainage routes, risk factors, and possible prognosis.

However, there are limitations to our current study. Firstly, although the sample size of this retrospective study was not small, there were only 37 cases of skip metastasis, which might lead to bias in the process of statistical analysis. For the same reason, we could not perform external validation to further validate our prediction model. Secondly, we did not further study each risk factor, especially the location of the tumor, which will be explained in more detail in our other studies. Thirdly, the present study lacks long-term follow-up and study on the prognosis of skip metastasis, and there are few studies which could be referred to at present. Therefore, we will conduct in-depth studies on the prognosis of skip metastasis in future studies in order to clarify the mechanism and mode of skip metastasis more completely.

\section{CONCLUSION}

There are significant differences between skip metastasis and other types of LNM in clinical characteristics, indicating that the lymph node drainage pathway of skip metastasis is different from

\section{REFERENCES}

1. So YK, Kim MJ, Kim S, Son YI. Lateral Lymph Node Metastasis in Papillary Thyroid Carcinoma: A Systematic Review and Meta-Analysis for Prevalence, Risk Factors, and Location. Int J Surg (2018) 50:94-103. doi: 10.1016/j.ijsu.2017.12.029

2. Liang J, Cai W, Feng D, Teng H, Mao F, Jiang Y, et al. Genetic Landscape of Papillary Thyroid Carcinoma in the Chinese Population. J Pathol (2018) 244:215-26. doi: 10.1002/path.5005

3. Heitz P, Moser H, Staub JJ. Thyroid Cancer: A Study of 573 Thyroid Tumors and 161 Autopsy Cases Observed Over a Thirty-Year Period. Cancer (1976) 37:2329-37. doi: 10.1002/1097-0142(197605)37:5<2329::AIDCNCR2820370523>3.0.CO;2-7

4. Davies L, Welch HG. Current Thyroid Cancer Trends in the United States. JAMA Otolaryngol Head Neck Surg (2014) 140:317-22. doi: 10.1001/ jamaoto.2014.1

5. Lin HW, Bhattacharyya N. Survival Impact of Treatment Options for Papillary Microcarcinoma of the Thyroid. Laryngoscope (2009) 119:1983-7. doi: $10.1002 /$ lary.20617 either CLNM or LLNM. Furthermore, we established a nomogram for predicting the risk of skip metastasis, which was able to effectively predict the present and potential risk of skip metastasis in patients without preoperative LNM clue.

\section{DATA AVAILABILITY STATEMENT}

The raw data supporting the conclusions of this article will be made available by the authors, without undue reservation.

\section{ETHICS STATEMENT}

Written informed consent was obtained from the individual(s) for the publication of any potentially identifiable images or data included in this article.

\section{AUTHOR CONTRIBUTIONS}

The article was written by ZY, YH, and QZ and they contributed equally to this work. WC, WQ, and LT provided guidance to the manuscript preparation. All authors contributed to the article and approved the submitted version.

\section{FUNDING}

This research was supported by the Shanghai Municipal Science and Technology Commission (19441905400) and Shanghai Jiaotong University (YG2019ZDA15).

\section{ACKNOWLEDGMENTS}

We thank all the authors who contributed to this topic. And thanks to all physicians for providing ultrasound tests and pathological diagnosis in this research.

6. Lee SH, Roh JL, Gong G, Cho KJ, Choi SH, Nam SY, et al. Risk Factors for Recurrence After Treatment of N1b Papillary Thyroid Carcinoma. Ann Surg (2019) 269:966-71. doi: 10.1097/SLA.0000000000002710

7. Albano D, Bertagna F, Bonacina M, Durmo R, Cerudelli E, Gazzilli M, et al Possible Delayed Diagnosis and Treatment of Metastatic Differentiated Thyroid Cancer by Adopting the 2015 ATA Guidelines. Eur J Endocrinol (2018) 179:143-51. doi: 10.1530/EJE-18-0253

8. Pandya A, Caoili EM, Jawad-Makki F, Wasnik AP, Shankar PR, Bude R, et al. Limitations of the 2015 ATA Guidelines for Prediction of Thyroid Cancer: A Review of 1947 Consecutive Aspirations. J Clin Endocrinol Metab (2018) 103:3496-502. doi: 10.1210/jc.2018-00792

9. Qubain SW, Nakano S, Baba M, Takao S, Aikou T. Distribution of Lymph Node Micrometastasis in Pn0 Well-Differentiated Thyroid Carcinoma. Surgery (2002) 131:249-56. doi: 10.1067/msy.2002.120657

10. Steck JH, Stabenow E, Bettoni GB, Steck S, Cernea CR. Accuracy of Sentinel Lymph Node Mapping in Detecting Occult Neck Metastasis in Papillary Thyroid Carcinoma. Arch Endocrinol Metab (2018) 62:296-302. doi: 10.20945/2359-3997000000038 
11. Yang Z, Heng Y, Lin J, Lu C, Yu D, Tao L, et al. Nomogram for Predicting Central Lymph Node Metastasis in Papillary Thyroid Cancer: A Retrospective Cohort Study of Two Clinical Centers. Cancer Res Treat (2020) 52:1010-8. doi: $10.4143 /$ crt.2020.254

12. Machens A, Hinze R, Thomusch O, Dralle H. Pattern of Nodal Metastasis for Primary and Reoperative Thyroid Cancer. World J Surg (2002) 26:22-8. doi: 10.1007/s00268-001-0176-3

13. Lee YS, Shin SC, Lim YS, Lee JC, Wang SG, Son SM, et al. Tumor LocationDependent Skip Lateral Cervical Lymph Node Metastasis in Papillary Thyroid Cancer. Head Neck (2014) 36:887-91. doi: 10.1002/hed.23391

14. Verburg FA, Mader U, Luster M, Reiners C. The Effects of the Union for International Cancer Control/American Joint Committee on Cancer Tumour, Node, Metastasis System Version 8 on Staging of Differentiated Thyroid Cancer: A Comparison to Version 7. Clin Endocrinol (Oxf) (2018) 88:950-6. doi: 10.1111/cen.13597

15. Lim YC, Koo BS. Predictive Factors of Skip Metastases to Lateral Neck Compartment Leaping Central Neck Compartment in Papillary Thyroid Carcinoma. Oral Oncol (2012) 48:262-5. doi: 10.1016/j.oraloncology. 2011.10.006

16. Zhao H, Huang T, Li H. Risk Factors for Skip Metastasis and Lateral Lymph Node Metastasis of Papillary Thyroid Cancer. Surgery (2019) 166:55-60. doi: 10.1016/j.surg.2019.01.025

17. Park JH, Lee YS, Kim BW, Chang HS, Park CS. Skip Lateral Neck Node Metastases in Papillary Thyroid Carcinoma. World J Surg (2012) 36:743-7. doi: 10.1007/s00268-012-1476-5

18. Ugurlu O, Baltaci S, Aslan G, Can C, Cal C, Elhan A, et al. Does Skip Metastasis or Other Lymph Node Parameters Have Additional Effects on Survival of Patients Undergoing Radical Cystectomy for Bladder Cancer? Korean J Urol (2015) 56:357-64. doi: 10.4111/kju.2015.56.5.357

19. Prenzel KL, Baldus SE, Monig SP, Tack D, Sinning JM, Gutschow CA, et al. Skip Metastasis in Nonsmall Cell Lung Carcinoma: Predictive Markers and Isolated Tumor Cells in N1 Lymph Nodes. Cancer (2004) 100:1909-17. doi: $10.1002 /$ cncr.20165

20. Liu J, Liu Q, Wang Y, Xia Z, Zhao G. Nodal Skip Metastasis is Associated With a Relatively Poor Prognosis in Thoracic Esophageal Squamous Cell Carcinoma. Eur J Surg Oncol (2016) 42:1202-5. doi: 10.1016/j.ejso. 2016.05.025

21. Hu D, Lin H, Zeng X, Wang T, Deng J, Su X. Risk Factors for and Prediction Model of Skip Metastasis to Lateral Lymph Nodes in Papillary Thyroid Carcinoma. World J Surg (2020) 44:1498-505. doi: 10.1007/s00268-01905332-0
22. Lei J, Zhong J, Jiang K, Li Z, Gong R, Zhu J. Skip Lateral Lymph Node Metastasis Leaping Over the Central Neck Compartment in Papillary Thyroid Carcinoma. Oncotarget (2017) 8:27022-33. doi: 10.18632/oncotarget.15388

23. Qiu Y, Fei Y, Liu J, Liu C, He X, Zhu N, et al. Prevalence, Risk Factors and Location of Skip Metastasis in Papillary Thyroid Carcinoma: A Systematic Review and Meta-Analysis. Cancer Manag Res (2019) 11:8721-30. doi: 10.2147/CMAR.S200628

24. Zhang TT, Qi XZ, Chen JP, Shi RL, Wen SS, Wang YL, et al. The Association Between Tumor's Location and Cervical Lymph Nodes Metastasis in Papillary Thyroid Cancer. Gland Surg (2019) 8:557-68. doi: 10.21037/gs.2019.10.02

25. Huang Z, Song M, Wang S, Huang J, Shi H, Huang Y, et al. Preoperative Serum Thyroglobulin Is a Risk Factor of Skip Metastasis in Papillary Thyroid Carcinoma. Ann Transl Med (2020) 8:389. doi: 10.21037/atm.2019.10.92

26. Dong W, Horiuchi K, Tokumitsu H, Sakamoto A, Noguchi E, Ueda Y, et al. Time-Varying Pattern of Mortality and Recurrence From Papillary Thyroid Cancer: Lessons From a Long-Term Follow-Up. Thyroid (2019) 29:802-8. doi: 10.1089/thy.2018.0128

27. Nam SH, Roh JL, Gong G, Cho KJ, Choi SH, Nam SY, et al. Nodal Factors Predictive of Recurrence After Thyroidectomy and Neck Dissection for Papillary Thyroid Carcinoma. Thyroid (2018) 28:88-95. doi: 10.1089/ thy.2017.0334

28. Wang F, Yu X, Shen X, Zhu G, Huang Y, Liu R, et al. The Prognostic Value of Tumor Multifocality in Clinical Outcomes of Papillary Thyroid Cancer. J Clin Endocrinol Metab (2017) 102:3241-50. doi: 10.1210/jc.2017-00277

Conflict of Interest: The authors declare that the research was conducted in the absence of any commercial or financial relationships that could be construed as a potential conflict of interest.

Publisher's Note: All claims expressed in this article are solely those of the authors and do not necessarily represent those of their affiliated organizations, or those of the publisher, the editors and the reviewers. Any product that may be evaluated in this article, or claim that may be made by its manufacturer, is not guaranteed or endorsed by the publisher.

Copyright (c) 2021 Yang, Heng, Zhao, Cao, Tao, Qiu and Cai. This is an open-access article distributed under the terms of the Creative Commons Attribution License (CC BY). The use, distribution or reproduction in other forums is permitted, provided the original author(s) and the copyright owner(s) are credited and that the original publication in this journal is cited, in accordance with accepted academic practice. No use, distribution or reproduction is permitted which does not comply with these terms. 